\title{
Digitalizing multidisciplinary pulmonary rehabilitation in COPD with a smartphone application: an international observational pilot study
}

This article was published in the following Dove Press journal:

International Journal of COPD

\author{
Frank Rassouli' \\ David Boutellier ${ }^{2}$ \\ Jonas Duss ${ }^{2}$ \\ Stephan Huber ${ }^{2}$ \\ Martin H Brutsche' \\ 'Lung Center, Department of Internal \\ Medicine, Cantonal Hospital St Gallen, \\ St Gallen, Switzerland; ${ }^{2}$ Kaia Health \\ $\mathrm{GmbH}$, Munich, Germany
}

Background: Concerning COPD, pulmonary rehabilitation (PR) has a positive effect on disease progression and mortality, is cost-effective, and is a part of recommendations of international guidelines. Only a minority of patients profit from conventional PR due to a lack of resources, physicians' guideline adherence, or patients' motivation. Novel digital therapies like Kaia COPD, a smartphone application that digitizes PR in COPD, are promising solutions to fill this void. Methods: Kaia COPD provides a digital version of PR and is certified as a class-I medical device in the European Union. We investigated anonymized data from users of the Kaia COPD app on inapp retention and the change in health-related quality of life (COPD assessment test and Chronic Respiratory Disease Questionnaire [CRQ]) during a period of 20 exercise days with the app.

Results: Of 349 app downloads, 56 users fulfilled inclusion criteria and 34 (61\%) had finished day 20 at the time of analysis and were included. Users took $33 \pm 11$ days to complete the 20 -day core program. Users finishing the program reduced their COPD assessment test scores (mean 2.5 units from $21.6 \pm 7.7$ to $19.1 \pm 8.4$ units, $P=0.008$ ). In finishers, there was a statistically significant effect above the minimum clinically important threshold of the CRQ score on the domains of fatigue, mastery, and emotional function. There was a statistically significant but not clinically relevant effect on the domain of dyspnea of CRQ.

Conclusion: Digitalizing PR with a smartphone app is feasible and accepted by selected patients. The app leads to short-term improvement of health-related quality of life in patients completing a 20-day core program. Due to its observational character, this study has several methodological limitations and was intended to show the feasibility and to extrapolate effect sizes for planned prospective randomized-controlled trials to confirm these findings.

Keywords: COPD, pulmonary rehabilitation, telehealth care, telerehabilitation

\section{Introduction}

COPD is a widespread disease with an estimated global prevalence of $11.7 \%$, which is characterized by persistent respiratory symptoms and a chronic obstructive airflow limitation, usually caused by chronic exposure to noxious inhaled agents. ${ }^{1}$ COPD is predicted to become the third leading cause of death and disability by 2030 , accounting for $8.6 \%$ of deaths worldwide, and the burden of chronic diseases linked to smoking, aging, and ambient air pollution will further increase. ${ }^{6}$ Due to the high prevalence, mortality, and morbidity, COPD will be one of the biggest public health challenges in the next century. ${ }^{7,8}$ To minimize the burden of COPD for the health care system, three cornerstones can be defined: smoking cessation, reduction of acute exacerbations,
Correspondence: Frank Rassouli Lung Center, Cantonal Hospital St Gallen, Rorschacher Strasse 95, CH-9007

St Gallen, Switzerland

Tel +4I 7| 4946020

Email frank.rassouli@kssg.ch 
and comorbidity management. ${ }^{7}$ Pulmonary rehabilitation (PR) combines these aspects in an integrative intervention in combination with muscle training.

Because respiratory and limb muscle dysfunction have been recognized as indicators for disease progression independent of lung function, ${ }^{9}$ physical exercise has become an indispensable measure to invert skeletal muscle dysfunction, ${ }^{10}$ improve exercise capacity and health-related quality of life (HRQoL), and reduce exacerbations.

PR has a positive effect on disease progression and mortality, is cost-effective, and is an important part of recommendations of international guidelines for symptomatic patients with COPD. However, even in spite of these recommendations, it often remains underutilized in clinical routine. ${ }^{11}$ Novel digital therapies have been found to be valid solutions to face this shortage. ${ }^{12}$

\section{Objectives}

The objective of this international observational pilot study is to measure the feasibility and effects of a digitalized PR program (Kaia COPD) delivered on a smartphone on HRQoL. The instruments used to accomplish this are two self-administered, validated questionnaires - the COPD Assessment Test (CAT) and the Chronic Respiratory Disease Questionnaire (CRQ)-SAS. ${ }^{13-16}$

\section{Methods}

\section{Study design and users}

This observational study analyzes user behavior and selfreported clinical parameters of COPD patients using the smartphone application Kaia COPD. The study cohort was recruited through the use of online channels in Germany, Austria, and Switzerland. These channels included COPD patient groups on Facebook as well as direct advertising through online media to specifically recruit COPD patients. Criteria for sign-up in the app were age 18 years or above, sufficient knowledge of German language, possession or access to a smartphone, and diagnosis of COPD by a physician (all self-reported in the app).

Criteria for inclusion in the study were Pro users of the app with full functionality and sign-up in the app in February and March 2018. For flow diagram of study collective, see Figure 1. Subscription costs for Pro users vary on the duration of the subscription package and range from $6.65 €$ to $11.66 €$.

Study recruitment took place between February and March 2018.

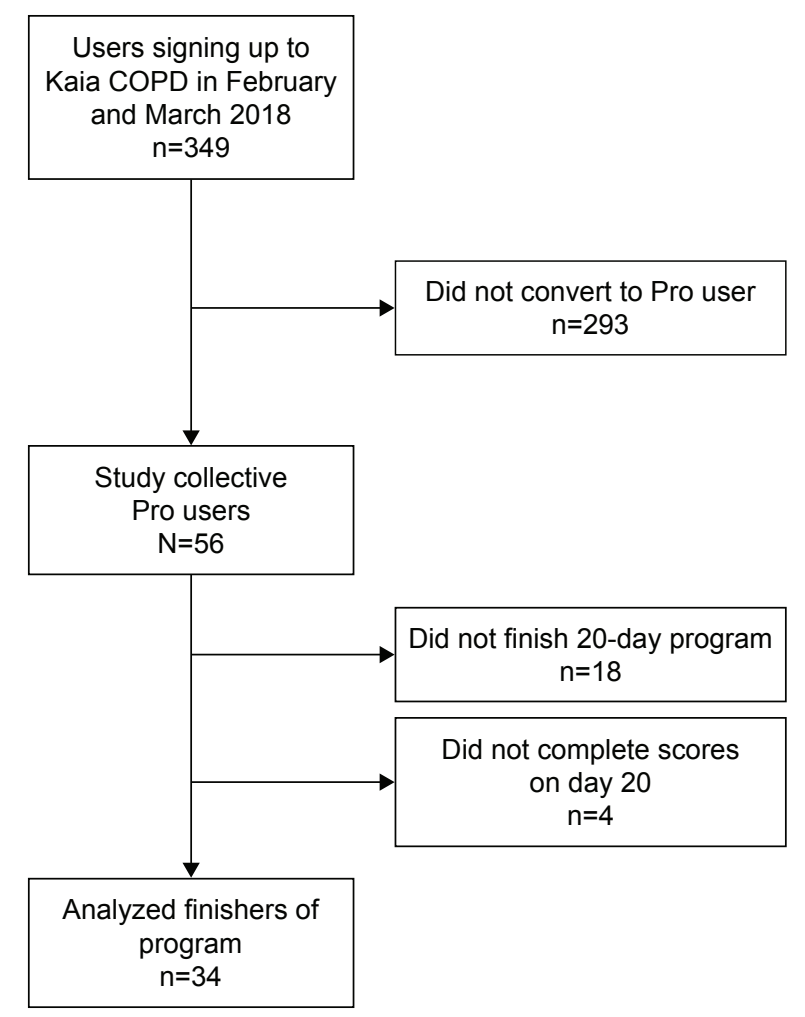

Figure I Flow diagram of the user database and study users.

All participants provided an electronic informed consent for the collection and analysis of data and agreed with the data privacy statement as well as the general terms and conditions of Kaia COPD. The study was approved by the ethical review board of the regional medical authorities (Bayerische Landesärztekammer) and received a waiver due to its retrospective and anonymized design (study number 2018-094). The study was carried out in accordance with the principles of the Declaration of Helsinki.

\section{Data collection}

Data were collected online from users of the app. For selfreported parameters, both the CAT and the CRQ were used. Patients were asked to fill out a survey both at the beginning of the study period and after having completed exercise sessions on 20 different days in the app. Datasets were anonymized before scientific evaluation.

\section{Statistical analysis}

Descriptive statistics were used for patient demographics (absolute or relative numbers). Differences in scores from baseline to the end-of-study visit were compared using paired $t$-tests. For the comparisons of intraindividual values at baseline vs follow-up, we used Student's $t$-tests. All variables 
Table I List of contraindications as mentioned in the app

\begin{tabular}{l} 
List of contraindications \\
- Acute myocardial infarction (within 2 days) \\
- Ongoing unstable angina or chest pain that comes irregularly \\
- Uncontrolled cardiac arrhythmia with hemodynamic compromise \\
- Active infection of your heart valves (endocarditis) \\
- Symptomatic tightening of your aortic valve (stenosis) \\
- Decompensated heart failure \\
- Acute pulmonary embolism, pulmonary infarction, or deep vein \\
- thrombosis \\
- Acute aortic dissection \\
- Active infection \\
- Unexplained other cardiovascular complaints (palpitations, \\
- Leg edema, cough) \\
\hline
\end{tabular}

were represented using mean and standard deviations. All statistical analyses were done using Microsoft Excel (version 16.13.1).

\section{Description of the app}

Kaia COPD is a multiplatform smartphone application developed by Kaia Health Software GmbH (Munich, Germany) and a CE-certified class I medical device in Europe. It is indicated for the self-management and exercise training for COPD. The software is available on the Apple App Store (iOS) and the Google Play Store. All users are being presented with a list of contraindications before signing up for the application (Table 1).

Each user's personal health status and physical limitations are evaluated in an integrated onboarding process. The app then provides daily exercises according to the resulting user profile from a selection of relevant disciplines in PR: psychology, physiotherapy, and patient education, as recommended by international medical guidelines. ${ }^{1-4}$ Furthermore, users have to confirm that they are physically able to conduct the exercises and that they have no contraindications to exercise therapy.

The daily PR exercises include the following elements: 1) physical exercises for strength and mobility training delivered in short videos, 2) patient education based on established patient guidelines, and 3) mindfulness techniques for disease coping. The user is presented with one exercise per category each day but can choose whether he or she wants to complete one, two, or all three elements. A screen shot of the app is provided in Figure 2.

The physical exercises were developed by the company in order to be suited for COPD patients, teachable via videos, and completed without additional training equipment than what is available in a common household (eg, chairs or water bottles). The Kaia algorithm tailors both the duration and the selection of the exercises to the specific user.

Patient education is based on the internationally validated patient guideline "Living well with COPD" and adapted to be delivered on a smartphone. Additional content, such as videos teaching the correct inhaler usage for all commonly used inhaler devices in the relevant countries, was added to the program when suitable. Patients can individually decide in a conversational interface about the depth of the content pieces. Disease progression is tracked daily with a feeling thermometer on a scale from 0 to 10 , a scale already verified for COPD, ${ }^{5}$ and with a dyspnea assessment on a linear Borg scale from 0 to 10 . These continuous measurements are
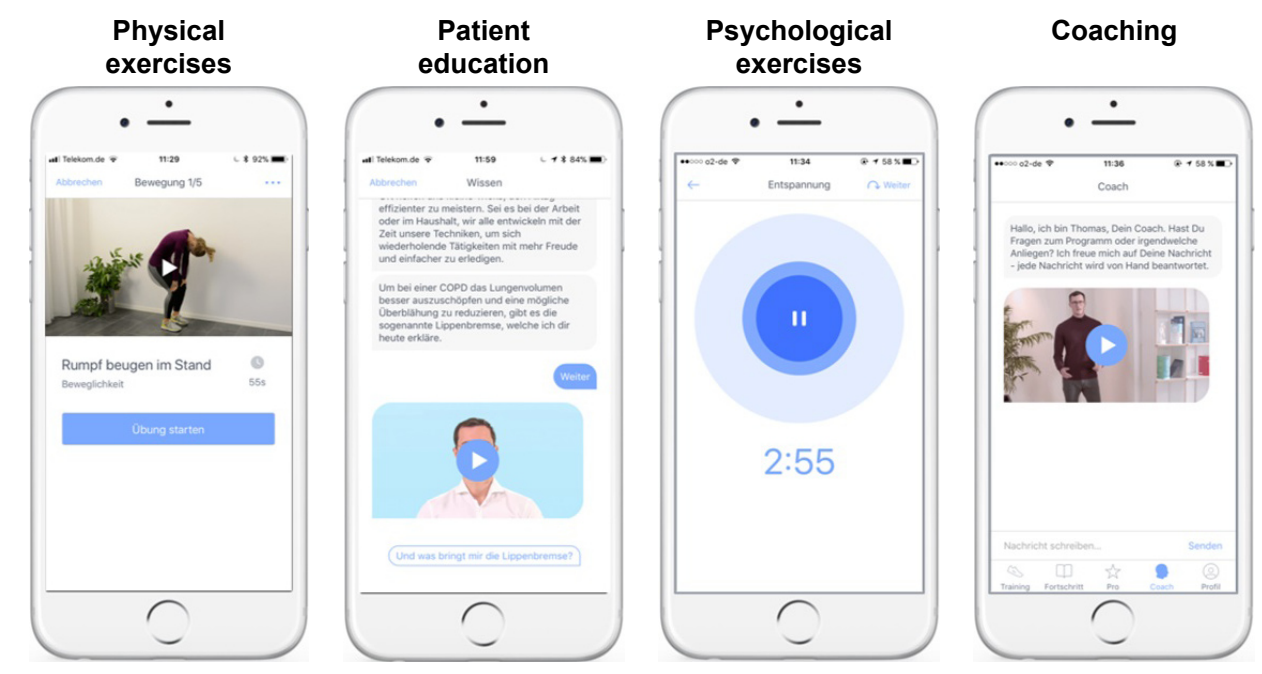

Figure 2 Screenshots of the Kaia COPD App. 
reported to the user in a dedicated screen together with the physical activity based on the number of steps per day as recorded by the smartphones integrated pedometer. Another function is an integrated coach chat in which patients can directly talk with a dedicated Kaia COPD coach for motivational and technical questions.

\section{Results}

\section{Patient demographics}

Of 349 app downloads, a total of 56 patients entered the PR program and were included. Participants were mostly female (80\%). The average CAT score was 22.4 ( \pm 7.6$)$ points, indicating a highly symptomatic patient population. Of these, $34(61 \%)$ finished the 20-day program and questionnaires and were included in the final analysis. Overall, there were no significant demographic differences between the initial sample including all users and participants who finished the program. For patient demographics, see Table 2.

\section{Effects of the 20-day program on self-reported CAT scores}

To assess the potential effects of a 20-day program of the Kaia COPD app on the CAT score, we compared baseline values with follow-up values in all patients where a follow-up score after completion of the 20-day program was available. In finishers, the CAT score declined from mean 21.6 \pm 7.7 units to $19.1 \pm 8.4$ units $(P=0.008$, Figure 3$)$.

\section{Effects of the 20-day program on self- reported CRQ scores}

To assess the potential effects of a 20-day program of the Kaia COPD app on the CRQ scores, we compared baseline values with follow-up values in all patients where a follow-up score after completion of the 20-day program was available. In finishers, there was a statistically significant effect above the minimum clinically important threshold of 0.5 points on the domains of fatigue (CRQ $3.4 \pm 1$ at baseline vs $4.1 \pm 1.3$ after 20 exercise days, $P<0.001$ ), mastery (CRQ $4.1 \pm 1.2$ at baseline vs $4.6 \pm 1.1$ after 20 exercise days, $P<0.001$ ), and emotional function (CRQ $3.8 \pm 1.2$ at baseline vs $4.4 \pm 1.4$ after 20 exercise days, $P<0.001)$. There was a statistically significant but not clinically relevant effect on the domain of dyspnea (CRQ 3.4 \pm 1 at baseline vs $3.2 \pm 1$ after 20 exercise days, $P<0.05)$ as the reduction remained below the minimum clinically important threshold (Figure 4).

\section{Discussion}

In this international observational pilot study, we assessed feasibility and short-term effects of the digitalized PR program “Kaia COPD," delivered on a smartphone, on HRQoL of severely symptomatic COPD patients. The low conversion rate from 349 sign-ups into 56 users is a common observation when using online channels to market health care apps (unpublished data of the authors). Roughly two-thirds of users completed a 20-day PR program as described above. We found a significant and clinically relevant improvement of the average CAT score and three out of four CRQ domains (fatigue, emotional function, and mastery) after completing the program.

Jones et al reported a mean improvement in CAT score of -2.2 points after completing a conventional PR program of 42 days duration. ${ }^{14}$ The observed reduction of -2.5 points after a 20-day digitalized PR program is, thus, a promising result for selected patients. The longitudinal course of the CAT is not only an important measure of HRQoL but is also directly linked to exacerbation risk, as shown previously by our group. ${ }^{17}$

A big meta-analysis on the effects of PR found significant and relevant improvements of all four domains of the CRQ, whereas we found comparable effects regarding fatigue, emotional function, and mastery, further supporting the usefulness of our intervention. ${ }^{18}$

Table 2 Demographic properties of all included patients and those who finished the 20-day program and completed the follow-up questionnaire

\begin{tabular}{|l|l|l|l|l|}
\hline & All users & $\begin{array}{l}\text { Finishers 20-day } \\
\text { program (baseline) }\end{array}$ & $\begin{array}{l}\text { Nonfinishers 20-day } \\
\text { program (baseline) }\end{array}$ & $\begin{array}{l}\boldsymbol{P} \text { (between finishers } \\
\text { and nonfinishers) }\end{array}$ \\
\hline Number $(\%)$ & 56 & $34(61)$ & $18(39)$ & \\
\hline Female & $45(80)$ & $26(76)$ & $15(83)$ & 0.560 \\
\hline Mean age (years) & $57.5( \pm 7.8)$ & $59.6( \pm 8.4)$ & $56.3( \pm 6.2)$ & 0.119 \\
\hline Mean height $(\mathrm{cm})$ & $166( \pm 8.7)$ & $167( \pm 9.3)$ & $166( \pm 8.7)$ & 0.526 \\
\hline Mean weight $(\mathrm{kg})$ & $73.5( \pm 24.6)$ & $82.2( \pm 28.9)$ & $74.8( \pm 15.9)$ & 0.306 \\
\hline Average CAT score & $22.4( \pm 7.6)$ & $21.6( \pm 7.6)$ & $24.5( \pm 7.3)$ & 0.167 \\
\hline
\end{tabular}

Note: $P$-values are nonpaired $t$-tests between groups.

Abbreviation: CAT, COPD assessment test. 


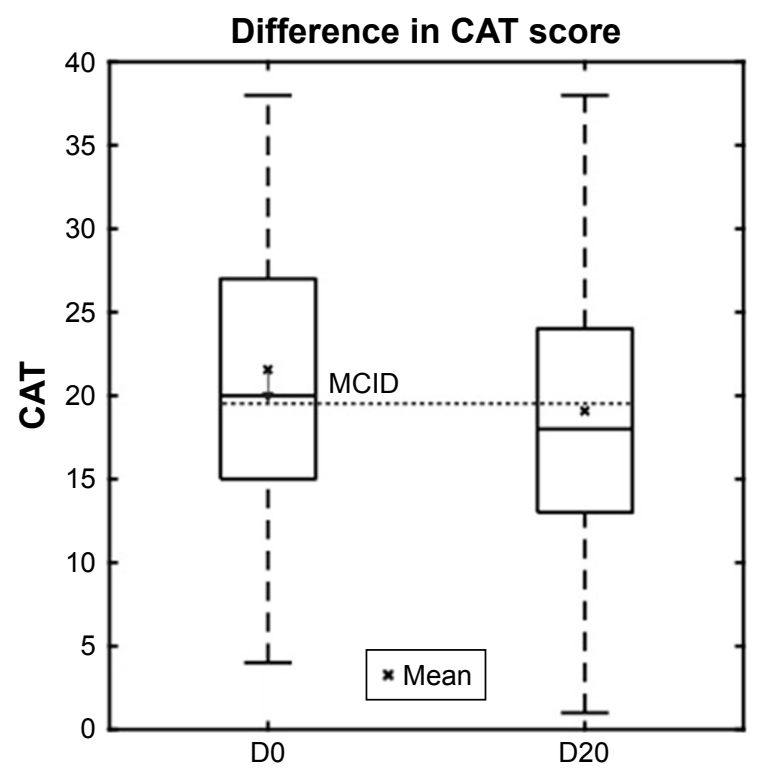

Figure 3 The box-plot shows CAT scores in patients finishing the program at baseline and after completion of the 20-day program.

Abbreviations: CAT, COPD assessment test; MCID, minimum clinically important difference.

A well-known problem is that the effects of PR decline after completing the program. ${ }^{19}$ Maintenance programs are able to preserve the effects. ${ }^{20,21}$ Our digitalized PR program could be an easily accessible solution to serve not only as an initial but also as a maintenance program, without burden of traveling for the patients.

Furthermore, it could offer a valid solution to face the initially mentioned problem of lack of PR capacities. ${ }^{11,12}$ In addition, it could serve as alternative for patients in remote areas. Of course, this study has limitations, mainly due to the small size, the observational pilot character, and a short duration of the intervention and potential selection bias. The longer-term effects remain to be demonstrated in future studies.

Furthermore, the study population was predominantly female, which does not reflect the usual demographic properties of COPD. Male patients are affected. This property of the study population is most likely caused by the channels with which patients were targeted and higher curiosity of female patients engage in digital self-management interventions. Similar observations have been made in scientific evaluations of self-management apps in pain medicine. ${ }^{22,23}$

Other limitations of the study mostly arise from its observational uncontrolled character. Only data of finishers could be collected, so there is a possible selection bias. Furthermore, there are only limited data available on participants' characteristics and the diagnosis was only self-confirmed during sign-up, which clearly limits the interpretation of the data. On the contrary, this is the first available study investigating the effects of digitalized PR with real-world data and showing its potential benefit that needs to be confirmed by further studies. Of note, the study was not intended to assess the clinical efficacy of the intervention but to show the potential feasibility and extrapolate effect sizes for planned prospective and controlled studies. These studies have to be conducted to eliminate the biases arising from the methodology of this study.

\section{Conclusion}

In conclusion, in this pilot study, we observed feasibility and promising effects of a 20-day digitalized PR program on CAT and HRQoL of severely symptomatic COPD patients, which has to be confirmed by well-designed randomizedcontrolled trials.

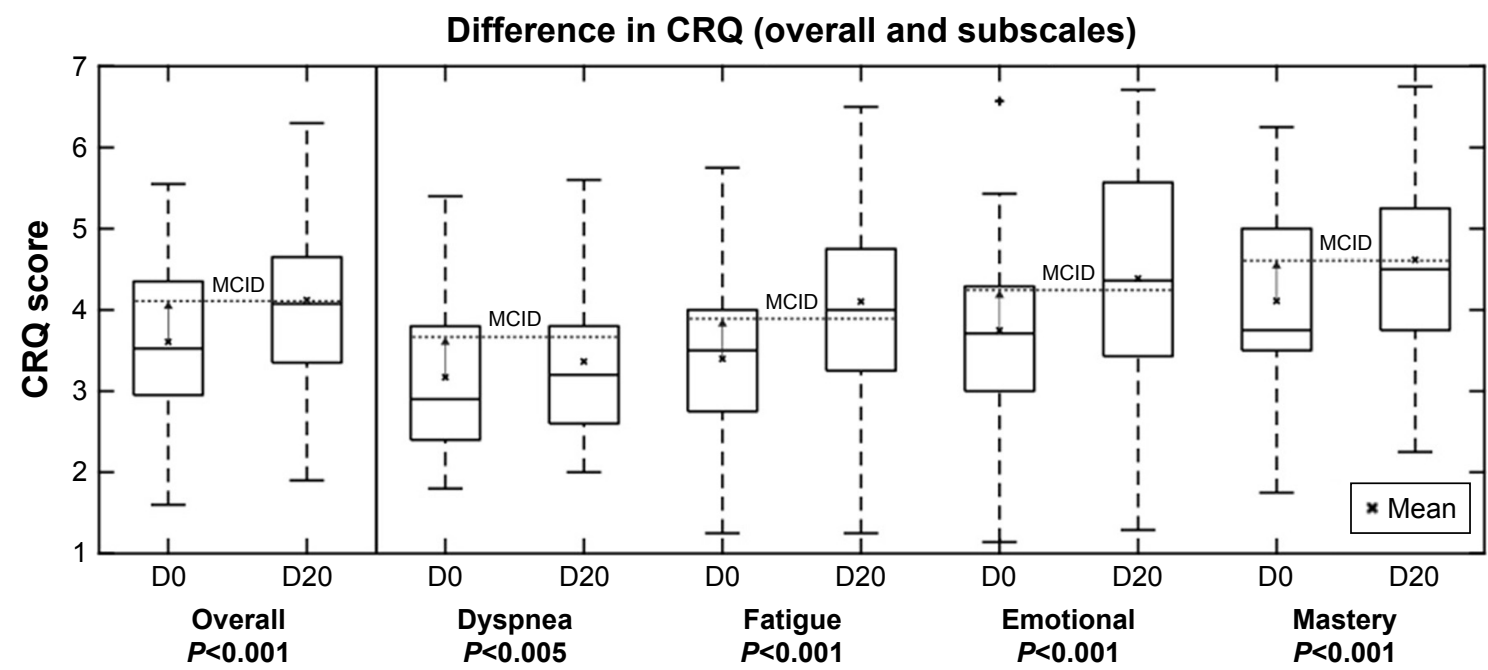

Figure 4 The box-plot shows CRQ scores in patients finishing the program at baseline (D0) and after completion (D20) of the 20-day program in the four domains: dyspnea, fatigue, emotional function, and mastery. Mean are shown with an $\mathrm{x}$ and the minimum clinically important difference for the CRQ score ( 0.5 change from baseline) is depicted. Abbreviations: CRQ, Chronic Respiratory Disease Questionnaire; MCID, minimum clinically important difference. 


\section{Disclosure}

The authors report no conflicts of interest in this work.

\section{References}

1. Global Initiative for Chronic Obstructive Lung Disease [webpage on the Internet]. Global strategy for the diagnosis, management, and prevention of chronic obstructive pulmonary disease; 2018. Available from: https:// goldcopd.org/wp-content/uploads/2017/11/GOLD-2018-v6.0-FINALrevised-20-Nov_WMS.pdf. Accessed November 18, 2018.

2. Pauwels RA, Buist AS, Calverley PM, Jenkins CR, Hurd SS. GOLD Scientific Committee. Global strategy for the diagnosis, management, and prevention of chronic obstructive pulmonary disease. NHLBI/ WHO Global Initiative for Chronic Obstructive Lung Disease (GOLD) Workshop Summary. Am J Respir Crit Care Med. 2001;163(5): 1256-1276.

3. Vestbo J, Prescott E, Lange P. Association of chronic mucus hypersecretion with FEV1 decline and chronic obstructive pulmonary disease morbidity. Copenhagen City Heart Study Group. Am J Respir Crit Care Med. 1996;153(5):1530-1535.

4. Burgel PR, Nesme-Meyer P, Chanez P, et al. Cough and sputum production are associated with frequent exacerbations and hospitalizations in COPD subjects. Chest. 2009;135(4):975-982.

5. Guerra S, Sherrill DL, Venker C, Ceccato CM, Halonen M, Martinez FD. Chronic bronchitis before age 50 years predicts incident airflow limitation and mortality risk. Thorax. 2009;64(10):894-900.

6. World Health Organization. World Health Statistics. Geneva: World Health Organanization; 2008;112.

7. López-Campos JL, Tan W, Soriano JB. Global burden of COPD. Respirology. 2016;21(1):14-23.

8. Casaburi R, Zuwallack R. Pulmonary rehabilitation for management of chronic obstructive pulmonary disease. N Engl J Med. 2009; 360(13):1329-1335.

9. Gea J, Pascual S, Casadevall C, Orozco-Levi M, Barreiro E. Muscle dysfunction in chronic obstructive pulmonary disease: update on causes and biological findings. $J$ Thorac Dis. 2015;7(10):E418-E438.

10. Puhan MA, Schünemann HJ, Frey M, Scharplatz M, Bachmann LM. How should COPD patients exercise during respiratory rehabilitation? Comparison of exercise modalities and intensities to treat skeletal muscle dysfunction. Thorax. 2005;60(5):367-375.

11. Gloeck1 R, Schneeberger T, Jarosch I, Kenn K. Pulmonary rehabilitation and exercise training in chronic obstructive pulmonary disease. Deutsches Aerzteblatt Online. 2018;115:117-124.
12. Bourne S, Devos R, North M, et al. Online versus face-to-face pulmonary rehabilitation for patients with chronic obstructive pulmonary disease: randomised controlled trial. BMJ Open. 2017;7(7):e014580.

13. Dodd JW, Hogg L, Nolan J, et al. The COPD assessment test (CAT): response to pulmonary rehabilitation. A multicentre, prospective study. Thorax. 2011;66(5):425-429.

14. Jones PW, Harding G, Wiklund I, et al. Tests of the responsiveness of the COPD assessment test following acute exacerbation and pulmonary rehabilitation. Chest. 2012;142(1):134-140.

15. Wijkstra PJ, Tenvergert EM, Van Altena R, et al. Reliability and validity of the chronic respiratory questionnaire (CRQ). Thorax. 1994;49(5): 465-467.

16. Williams JE, Singh SJ, Sewell L, Guyatt GH, Morgan MD. Development of a self-reported Chronic Respiratory Questionnaire (CRQ-SR). Thorax. 2001;56(12):954-959.

17. Rassouli F, Baty F, Stolz D. Longitudinal change of COPD assessment test (CAT) in a telehealthcare cohort is associated with exacerbation risk. Int J Chron Obstruct Pulmon Dis. 2017;24(12):3103-3109.

18. McCarthy B, Casey D, Devane D, Murphy K, Murphy E, Lacasse Y. Pulmonary rehabilitation for chronic obstructive pulmonary disease. Cochrane Database Syst Rev. 2015;(1):CD003793.

19. Spruit MA, Singh SJ, Garvey C, et al; ATS/ERS Task Force on Pulmonary Rehabilitation. An official American Thoracic Society/European Respiratory Society statement: key concepts and advances in pulmonary rehabilitation. Am J Respir Crit Care Med. 2013;188(8):e13-e64.

20. Ringbaek T, Brøndum E, Martinez G, Lange P; Pulmonary Rehabilitation Research Group. Rehabilitation in COPD: the long-term effect of a supervised 7-week program succeeded by a self-monitored walking program. Chron Respir Dis. 2008;5(2):75-80.

21. Hill K, Bansal V, Brooks D, Goldstein RS. Repeat pulmonary rehabilitation programs confer similar increases in functional exercise capacity to initial programs. J Cardiopulm Rehabil Prev. 2008;28(6):410-414.

22. Huber S, Priebe JA, Baumann KM, Plidschun A, Schiessl C, Tölle TR. Treatment of low back pain with a digital multidisciplinary pain treatment app: short-term results. JMIR Rehabil Assist Technol. 2017; 4(2):e11.

23. Nicholl BI, Sandal LF, Stochkendahl MJ, et al. Digital support interventions for the self-management of low back pain: a systematic review. J Med Internet Res. 2017;19(5):e179.
International Journal of COPD

\section{Publish your work in this journal}

The International Journal of COPD is an international, peer-reviewed journal of therapeutics and pharmacology focusing on concise rapid reporting of clinical studies and reviews in COPD. Special focus is given to the pathophysiological processes underlying the disease, intervention programs, patient focused education, and self management protocols.

\section{Dovepress}

This journal is indexed on PubMed Central, MedLine and CAS. The manuscript management system is completely online and includes a very quick and fair peer-review system, which is all easy to use. Visit $\mathrm{http}: / / \mathrm{www}$.dovepress.com/testimonials.php to read real quotes from published authors. 